\title{
(2) OPEN ACCESS \\ Papilloplex HR-HPV test has non-inferior clinical performance for detection of human papillomavirus infection: assessment using the VALGENT framework
}

\author{
Ramya Bhatia 이 , 1,2 Elia Alcaniz Boada, ${ }^{2}$ Jesper Hansen Bonde, ${ }^{3}$ Wim G V Quint, ${ }^{4}$ \\ Lan $\mathrm{Xu}_{1}{ }^{5}$ Ditte Moller Ejegod, ${ }^{6}$ Kate Cuschieri, ${ }^{1}$ Marc Arbyn $^{5}$
}

'Laboratory Medicine, SHPVRL, NHS Lothian, Edinburgh, UK ${ }^{2}$ HPV Research Group, University of Edinburgh Centre for Reproductive Biology, Edinburgh, UK

${ }^{3}$ Department of Pathology, Copenhagen University Hospital, Copehnagen, Denmark ${ }^{4}$ Delft Diagnostic Laboratory (DDL), Rijswijk, The Netherlands ${ }^{5}$ Belgian Cancer Centre,

Sciensano, Brussel, Belgium ${ }^{6}$ Hvidovre Hospital, Hvidovre, Denmark

Correspondence to Dr Ramya Bhatia, Scottish HPV Reference Laboratory, NHS Lothian, Edinburgh, UK; ramya. bhatia@ed.ac.uk

Received 29 July 2021 Accepted 5 September 2021

D Check for updates

(c) Author(s) (or their employer(s)) 2021. Re-use permitted under CC BY-NC. No commercial re-use. See rights and permissions. Published by BMJ.

To cite: Bhatia $\mathrm{R}$, Alcaniz Boada E, Bonde JH, et al. $J$ Clin Pathol Epub ahead of print: [please include Day Month Year]. doi:10.1136/ jclinpath-2021-207864

\section{ABSTRACT}

Aim The Papilloplex high-risk human papillomavirus (hrHPV) test (Genefirst, Oxford, UK) is a single tube real-time HPV test which provides multiplex detection and separate identification of $14 \mathrm{hrHPV}$ types. Here, we present the clinical validation of the test in SurePath samples in comparison to a clinically validated reference test, the GP5+/6+Enzyme ImmunoAssay (GP5+/6+EIA) using the VALGENT (VALidation of HPV GENotyping Tests) framework.

Methods Clinical performance was assessed using 998 unselected, cervical screening samples enriched with 297 cytologically abnormal specimens (100 atypical squamous cells of unspecified significance, 100 lowgrade squamous intraepithelial lesions, 97 high-grade squamous intraepithelial lesions). Cases were defined as women diagnosed with histologically confirmed cervical intraepithelial neoplasia two or more ( $\geq \mathrm{CIN2}, \mathrm{N}=119$ ) and controls defined as women with two subsequent negative cytology results ( $N=834)$.

Results The Papilloplex HR-HPV test has non-inferior sensitivity for detection of cervical precancer $(p=0.0001$ for $\geq$ CIN2 and $p=0.0005$ for $\geq$ CIN3) and non-inferior specificity, compared with GP5+/6+EIA (pni=0.0167)). The assay also showed excellent or good agreement for overall hrHPV and nearly all individual HPV types as compared with GP5+/6+EIA/Luminex.

Conclusion The Papilloplex HR-HPV applied on cervical specimens stored in SurePath medium fulfils the international clinical accuracy criteria for use in cervical cancer screening.

\section{INTRODUCTION}

Detection of high-risk human papillomavirus (hrHPV) in cervical specimens is becoming the mainstay test used in cervical screening because it is more consistent and effective than cervical cytology ${ }^{12}$ and visual inspection. ${ }^{3}$ Currently, there are over 200 different assays that detect nucleic acids of HPV, but relatively few are clinically validated. ${ }^{4-6}$ Only clinically validated hrHPV, that assure an optimal balance in sensitivity and specificity for detecting cervical precancer should be used in screening. ${ }^{78}$

The Papilloplex HR-HPV test (Genefirst, Oxford, UK) is a single tube real-time HPV test which provides multiplex detection and separate identification of $14 \mathrm{hrHPV}$ types 16, 18, 31, 33, 35, $39,45,51,52,56,58,59,66$ and 68 together with a human control target, all reported individually.
The assay is based on multiplex probe amplification (MPA) technology and uses differing meltingcurve profiles to allow the differentiation of up to six targets per fluorescence channel of a real-time PCR reaction ${ }^{9}$ allowing for extended genotyping information.

The assay has previously shown good analytical sensitivity and specificity and reproducibility for the 14 hrHPV types. ${ }^{10}$ Preliminary clinical performance studies, using archived cervical Thinprep samples, demonstrated similar performance of Papilloplex for detection of cervical intraepithelial neoplasia grade 2 ( $\geq$ CIN2) to that of two clinically validated HPV tests: RealTime hrHPV test (RealTime) and Hybrid Capture 2 HPV test (HC2), along with high agreement for HR-HPV and individual genotypes when compared with Linear Array (Roche) and Optiplex HPV test (Diamex). ${ }^{11}$ However, while encouraging, the data described above derived using a convenience sample rather than on a welldefined set of specimens that would enable clinical validation according to internationally recognised criteria.

To address this, the clinical performance of the Papilloplex HR-HPV test was assessed according to the international guidelines of Meijer et al ${ }^{12}$ using the VALidation of HPV GENotyping Tests (VALGENT) framework. ${ }^{13} 14$ Among other things, VALGENT provides a 'fast track' to validation by the international guidelines by using diseaseenriched sample panels, previously characterised for HPV status and cytopathological information. A total of four iterations of VALGENT have been performed to date. Valgent 2 and 3 (V2-V3) supported assay evaluations in ThinPrep media (Hologic, Bedford, Massachusetts, USA) ${ }^{15-23}$ while the first and the fourth iteration supported assay evaluations in SurePath (Becton Dickinson (BD), USA). ${ }^{24}$

Papilloplex is part of the fourth iteration of VALGENT ${ }^{14}$ comprising eleven different HPV genotyping assays from eight different manufacturers, using GP5+/6 + PCR enzyme immunoassay (EIA) as comparator ${ }^{14}$ and SurePath collected cervical screening samples from an organised screening programme. Assays interrogated include those with extended genotyping capability such as Onclarity HPV assay (Becton Dickinson, New Jersey, USA), ${ }^{15}$ limited genotyping such as Cobas 4800 HPV assay (Roche, Basel, USA), ${ }^{25}$ Harmonia HPV (Liferiver, Shanghai, China), ${ }^{26}$ HPV-risk 
assay (Self-Screen BV, Amsterdam, The Netherlands) ${ }^{18} 27$ and those with full genotyping abilities-CLART HPV4 assay (Genomica, Madrid, Spain), HPV MassArray assay (Agena Bioscience, Hamburg, Germany), INNO-LiPA Genotyping Extra II test (FujiriBio Europe,Ghent, Belgium), Venus HPV (Liferiver, Shanghai, China) and Papilloplex HPV assay (Genefirst, Oxford, UK). ${ }^{102125}$ The Papilloplex HPV assay is validated analytically on different extraction systems (including NucliSENS easyMAG and QIAamp DNA Mini Kit) and on a commonly used PCR machine (Applied Biosystems 7500) and provides individual genotyping results with no post-PCR steps.

In the current paper, we further explore clinical performance by determining whether its accuracy to detect cervical precancer is non inferior to a standard, clinically validated comparator HPV test (HPV GP5 +/6+PCR EIA).

\section{MATERIALS AND METHODS}

\section{Study population and processing}

The VALGENT4 panel consists of 998 unselected, consecutive routine samples collected from women aged 30-59 from the Danish cervical cancer screening programme enriched with 297 cytologically abnormal specimens (100 atypical squamous cells of unspecified significance, 100 low-grade squamous intraepithelial lesions, 97 high-grade squamous intraepithelial lesions). A detailed description of the study population, sample collection and processing can be found in Bonde et al. ${ }^{14}$ Samples were collected at the Molecular Pathology Laboratory, Department of Pathology, Copenhagen University Hospital, Hvidovre and DNA was extracted using MagNA Pure96 system (Roche Diagnostics, Pleasanton, California, USA). ${ }^{14}$ In brief, four extractions were carried out using $1 \mathrm{~mL}$ sample each and the DNA eluates were pooled to get a $400 \mu \mathrm{L}$ eluate. DNA was extracted in 2017 and an aliquot of DNA was sent to the HPV Research Group, University of Edinburgh, UK, to perform Papilloplex testing and to Delft Diagnostic Laboratories (DDL), Rijswijk, The Netherlands, for GP5 +/6+EIA test. Papilloplex testing was carried out in 2018 and GP5+/6+EIA test was subsequently performed and completed on the GP5+/6+amplicons in 2018.

Screening and outcome history (average follow-up of 33 months (min 32, $\max 35$ months)) was retrieved from the Danish Pathology Data Bank (PatoBank). All clinical follow-up was managed according to Danish guidelines, and the result of the VALGENT HPV test evaluations did not affect clinical follow-up recommendations. Samples associated with $\geq$ CIN2 or worse were considered 'cases' and used for the evaluation of sensitivity.

In total, 122 cases with precancer were detected in VALGENT-4 which included 83 with CIN3 or worse ( $\geq$ CIN3). Controls were defined based on two consecutive negative cytology results (at enrolment and at 12-24 months before enrolment) and used for specificity assessment $(2 \mathrm{x}$ negative for intraepithelial lesion or malignancy (NILM)). In the final analysable cohort (after removing invalids in Papilloplex), there were 834 controls and 119 cases (including $82 \geq \mathrm{CIN} 3$ ) (figure 1 ).

\section{Papilloplex HR-HPV test}

Papilloplex HR-HPV test (Genefirst, Oxford, UK) is a CE marked multiplex real time PCR test targeting the L1 region for the detection of $14 \mathrm{hrHPV}$ types $(16,18,31,33,35,39,45$, $51,52,56,58,59,66$ and 68) individually. The test includes an internal control (IC) targeting ARHGEF11 gene. The targets covered by different fluorophore channels are as follows:

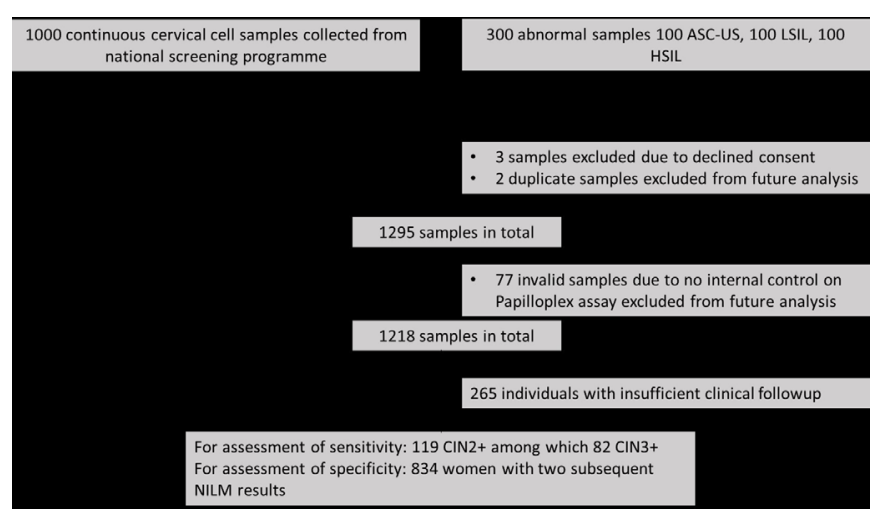

Figure 1 Flow chart representing the study population. A total of $119 \geq$ CIN2 and $82 \geq$ CIN3 samples were analysed. ASC-US, atypical squamous cells of unspecified significance; CIN2, cervical intraepithelial neoplasia grade 2; HSIL, high-grade squamous intraepithelial lesions; LSIL, low-grade squamous intraepithelial lesions; NILM, negative for intraepithelial lesion or malignancy.

FAM- HPV16, 18, 31, 52, 59; HEX-39, 58, 68; ROX- 33, 35, 45, 51, 56, 66; Cy5-IC.

Testing was performed according to the instructions for use (IFU-P0111v3.0, May 2017). In brief, $4 \mu \mathrm{L}$ of sample DNA was added to a total of $20 \mu \mathrm{L}$ reaction mix. PCR was performed using the ABI7500 Real Time PCR System and amplification analysis using Applied Biosystems Sequence Detection Software V.1.4-7500 Fast System SDS software. Baseline settings for the assay were modified from the recommended IFU settings on discussion with the manufacturer to relative fluorescence values for different channels set as follows-Cy5-10 000, FAM-40 000, Hex-10 000 and ROX-40 000 (IFU recommendation was Cy5-50, 000, FAM-100 000, Hex-25 000 and ROX-100 000). Melting profile analysis and report creation was carried out using GeneFirst MPA Analysis Software, version 0.5. The PCR protocol takes approximately 2.5 hours. Testing was performed in batches along with kit controls. Baseline and threshold for the real time amplification curves were standardised between runs. Samples were considered valid if a CT value of $\leq 38$ was obtained for IC and positive if the CT value was $\leq 36$ for the HPV channels. Samples were deemed invalid if IC CT value was $>38$ and no HPV type was detected in the sample. Any samples that were invalid were repeated once.

\section{Standard HPV comparator test GP5+/6+ EIA}

GP5+/6+PCR EIA which detects the 14 hrHPV types $(16,18$, $31,33,35,39,45,51,52,56,58,59,66$ and 68) was used as the standard HPV comparator test to assess and compare the clinical accuracy of the Papilloplex HR-HPV test. ${ }^{12}$ Testing was performed according to manufacturer's instructions.

\section{Assessment of clinical performance and non-inferiority to comparator test}

HrHPV positivity was based on presence of at least one of the defined $14 \mathrm{hrHPV}$ types. Sensitivity and specificity of Papilloplex were compared with GP5+/6+EIA. The McNemar $\chi^{2}$ test was applied to assess differences between matched proportions $\left(\mathrm{P}_{\mathrm{McN}}\right)$. A matched non-inferiority $\left(\mathrm{p}_{\mathrm{ni}}\right)$ test was performed with a 0.90 relative sensitivity threshold and 0.98 relative specificity threshold. A p value level of 0.05 was set for significance for both $\mathrm{p}_{\mathrm{McN}}$ and $\mathrm{p}_{\mathrm{ni}}{ }^{28}$ All the analyses were carried out using STATA V.14. Data analysis was performed at the Unit of Cancer 
Table 1 Prevalence of HPV types detected by Papilloplex HR-HPV test in the VALGENT-4 screening cohort

\begin{tabular}{lrll}
\hline HPV type & $\mathbf{N}$ & $\%$ & $95 \%$ Cl \\
\hline hrHPV & 140 & 15.1 & 12.9 to 17.6 \\
\hline HPV16 & 17 & 1.8 & 1.2 to 2.9 \\
\hline HPV18 & 8 & 0.9 & 0.4 to 16.9 \\
\hline HPV31 & 13 & 1.4 & 0.8 to 2.4 \\
\hline HPV33 & 8 & 0.9 & 0.4 to 16.9 \\
\hline HPV35 & 2 & 0.2 & 0.1 to 0.8 \\
\hline HPV39 & 5 & 0.5 & 0.2 to 1.3 \\
\hline HPV45 & 24 & 2.6 & 1.8 to 3.8 \\
\hline HPV51 & 14 & 1.5 & 0.9 to 2.5 \\
\hline HPV52 & 15 & 1.6 & 1 to 2.7 \\
\hline HPV56 & 6 & 0.7 & 0.3 to 1.4 \\
\hline HPV58 & 16 & 1.7 & 1.1 to 2.8 \\
\hline HPV59 & 4 & 0.4 & 0.2 to 1.1 \\
\hline HPV66 & 23 & 2.5 & 1.7 to 3.7 \\
\hline HPV68 & 11 & 1.2 & 0.7 to 2.1 \\
\hline
\end{tabular}

hrHPV, high-risk human papillomavirus; VALGENT-4, VALidation of HPV GENotyping Tests 4.

Epidemiology (Sciensano, Belgian Cancer Centre) in Brussels, Belgium.

\section{RESULTS}

\section{Hpv prevalence}

All samples $(n=1295)$ tested were valid for GP5+/6+EIA but 77 (5.9\%) samples were invalid for Papilloplex as defined by a cut-off of $\geq 38$ for IC with no HPV type detected (figure 1). HrHPV prevalence with the Papilloplex test in the screening cohort $(\mathrm{n}=927)$ was $15.1 \%$ (95\% CI: $12.9 \%$ to $17.6 \%)$, while prevalence of the comparator assay, GP5+/6+EIA was $14.3 \%$. Prevalence of individual HPV types detected by Papilloplex test ranged from $0.2 \%$ (HPV35) to 2.59\% (HPV45) (table 1).

\section{Overall hrHPV and type specific agreement between Papilloplex HR-HPV and GP5+/6+ EIA and GP5+/6+ Luminex, respectively}

Table 2 shows the overall concordance for hrHPV (14 hrHPV types) between Papilloplex, HPV-HPV GP5+/6+EIA and the type specific concordance between Papilloplex HPV-HPV GP5+/6+-Luminex). Overall concordance of the two assays for $14 \mathrm{hrHPV}$ types was $92.4 \%(\kappa=0.727)$, when compared with GP5+/6+EIA and 92.1\% (0.823) when compared with GP5 +/6+ (Luminex) LMNX, indicating excellent agreement. The level of agreement was also excellent for detection of HPV16 $(\mathrm{k}=0.867)$, HPV31 $(\mathrm{k}=0.903)$, HPV33 $(\mathrm{k}=0.809)$, HPV39 $(k=1.000)$, HPV45 $(k=0.802)$ and HPV66 $(k=0.811)$. For all other HPV types the level of agreement was good except HPV59 which had moderate agreement between the two assays.

\section{Absolute sensitivity and specificity of Papilloplex HR-HPV test} Papilloplex detected 114 of $119 \geq$ CIN2 cases $(95.8 \%$, 95\% CI: $0.91 \%$ to $0.99 \%)$ and 80 of the $82 \geq \mathrm{CIN} 3$ cases $(97.6 \%, 95 \%$ CI: $0.92 \%$ to $1.00 \%)$. Absolute specificity for the $2 \mathrm{x}$ NILM cohort for $\leq$ CIN1 was $89.1 \%$ (95\% CI: $0.87 \%$ to $0.91 \%$ ).

\section{Sensitivity and specificity of Papilloplex HR-HPV test when compared with GP5+/6+-EIA}

Relative sensitivity and specificity can be seen in table 3. Papilloplex HR-HPV test has non-inferior sensitivity and specificity to
Table 2 Type specific concordance between Papilloplex and GP5+/6+PCR-EIA/Luminex

\begin{tabular}{lccrrrl}
\hline HPV type & P+/G+ & P+/G- & P-/G+ & P-/G- & Concordance & K \\
\hline 14hrHPV* & 343 & 48 & 43 & 766 & $92.4 \%$ & 0.827 \\
14hrHPV+ & 349 & 42 & 52 & 752 & $92.1 \%$ & 0.823 \\
HPV16 & 78 & 0 & 22 & 1095 & $98.2 \%$ & 0.867 \\
HPV18 & 23 & 0 & 25 & 1147 & $97.9 \%$ & 0.639 \\
HPV31 & 44 & 3 & 6 & 1142 & $99.3 \%$ & 0.903 \\
HPV33 & 24 & 1 & 10 & 1160 & $99.1 \%$ & 0.809 \\
HPV35 & 10 & 1 & 11 & 1173 & $99.0 \%$ & 0.620 \\
HPV39 & 16 & 0 & 0 & 1179 & $100.0 \%$ & 1.000 \\
HPV45 & 36 & 11 & 6 & 1142 & $98.6 \%$ & 0.802 \\
HPV51 & 23 & 10 & 14 & 1148 & $98.0 \%$ & 0.647 \\
HPV52 & 30 & 15 & 2 & 1148 & $98.6 \%$ & 0.772 \\
HPV56 & 28 & 1 & 15 & 1151 & $98.7 \%$ & 0.771 \\
HPV58 & 20 & 9 & 1 & 1165 & $99.1 \%$ & 0.796 \\
HPV59 & 9 & 5 & 12 & 1169 & $98.6 \%$ & 0.507 \\
HPV66 & 36 & 10 & 6 & 1143 & $98.7 \%$ & 0.811 \\
HPV68 & 12 & 8 & 0 & 1175 & $99.3 \%$ & 0.747 \\
\hline 'P'
\end{tabular}

'P'=Papilloplex HR-HPV test and 'G=GP5+/6+-Luminex'. Colour legend (adapted from Landis and Koch for the levels of agreement $(31,12))$ : dark green $(1.00$ $\geq \kappa \geq 0.80)$ : excellent; light green $(0.80 \geq \kappa>0.60)$ : good; yellow $(0.60 \geq \kappa>0.40)$ : moderate; orange $(0.40 \geq \kappa>0.20)$ : fair; red $(0.20 \geq \kappa>0.00)$ : poor

${ }^{*}$ Concordance between Papilloplex and GP5+/6+PCR EIA for presence or absence of the 14 hrHPV types.

tConcordance between Papilloplex and GP5+/6+PCR-LMNX.

EIA, enzyme immunoassay; hrHPV, high-risk human papillomavirus.

GP5 + $/ 6+$ EIA for detection of $\geq$ CIN2 and $\geq$ CIN3 $(p=0.0001$ for $\geq$ CIN2 and $p=0.0005$ for $\geq$ CIN3). Of the $119 \geq \mathrm{CIN} 2$ cases, Papilloplex detected 114 and GP5+/6+EIA detected 112 cases. Of the $82 \geq \mathrm{CIN} 3$ cases, Papilloplex identified 80 cases while GP5 + $/ 6+$ EIA detected 78 cases. The specificity of Papilloplex was also non-inferior to GP5 +/6+EIA (relative specificity: 1.003 (95\% CI: 0.98 to $\left.1.02, \mathrm{p}_{\mathrm{ni}}=0.0167\right)$ ).

\section{DISCUSSION}

Papilloplex HR-HPV assay is a PCR based HPV test that detects 14 hrHPVs individually. Based on a simple and rapid ( $<3$ hours) real-time PCR system, the assay is validated on different extraction systems (including NucliSENS easyMAG and QIAamp DNA Mini Kit) and on a commonly used PCR machine (Applied Biosystems 7500) offering flexibility to laboratories using the assay. The ability to provide individual genotyping within a single closed tube assay offers advantages over other genotyping assays in reducing reaction times, number of steps required for assay setup and in reducing contamination related to multiple reactions. The assay was set up manually in this study and analysis was performed using two softwares. However, work around automation of the setup of PCR reactions and streamlining data analysis which will improve the throughput and ease of use of the assay for large scale screening is currently being undertaken by the manufacturer.

In this study, we had an invalidity rate, where samples were IC negative (CT value $>38$ ) and negative for HR-HPV types (CT value $>38$ ), of $5.8 \%$. This is substantially above what has recently been reported for HPV-based cervical screening. ${ }^{29}$ Increasing the IC CT value cut-off to $\geq 39, \geq 40$ or $\geq 41$ reduced the invalidity rate to $4.9 \%, 4.5 \%$ and $4.2 \%$. However, some technical reasons might have contributed to the high invalidity rate, including issues with the PCR mix reagents reacting to certain plastics (PCR tubes vs PCR plates and type of plate holders) on the ABI7500, requiring a change of 


\begin{tabular}{|c|c|c|c|c|c|c|c|}
\hline & \multirow{2}{*}{$\begin{array}{l}\text { Papilloplex } \\
\text { HR HPV }\end{array}$} & \multicolumn{3}{|l|}{ Gp5+/6+ } & \multirow{2}{*}{$\begin{array}{l}\text { Relative } \\
\text { accuracy }\end{array}$} & \multirow[b]{2}{*}{$\mathbf{P}_{\mathrm{MCN}}$} & \multirow[b]{2}{*}{$P_{n i}$} \\
\hline & & Positive & Negative & Total & & & \\
\hline \multirow{3}{*}{$\begin{array}{l}\text { Sensitivity } \\
\geq \text { CIN2 }\end{array}$} & Positive & 111 & 3 & 114 & \multirow{3}{*}{$\begin{array}{l}1.018 \\
(0.98-1.05)\end{array}$} & \multirow[t]{3}{*}{0.625} & \multirow[t]{3}{*}{0.0001} \\
\hline & Negative & 1 & 4 & 5 & & & \\
\hline & Total & 112 & 7 & 119 & & & \\
\hline \multirow{3}{*}{$\begin{array}{l}\text { Sensitivity } \\
\geq \text { CIN3 }\end{array}$} & Positive & 78 & 2 & 80 & \multirow{3}{*}{$\begin{array}{l}1.026 \\
(0.99-1.06)\end{array}$} & \multirow[t]{3}{*}{0.500} & \multirow[t]{3}{*}{0.0005} \\
\hline & Negative & 0 & 2 & 2 & & & \\
\hline & Total & 78 & 4 & 82 & & & \\
\hline \multirow{3}{*}{$\begin{array}{l}\text { Specificity } \\
\text { SCIN1 }\end{array}$} & Positive & 62 & 29 & 91 & \multirow{3}{*}{$\begin{array}{l}1.003 \\
(0.98-1.02)\end{array}$} & \multirow[t]{3}{*}{0.897} & \multirow[t]{3}{*}{0.0167} \\
\hline & Negative & 31 & 712 & 743 & & & \\
\hline & Total & 93 & 741 & 834 & & & \\
\hline
\end{tabular}

Sensitivity for histologically confirmed $\geq \operatorname{CIN2}(n=119)$ or $\geq \operatorname{CIN} 3$ ( $n=82$ ), specificity for $\leq$ CIN1 considering women with two consecutive negative cytology (at enrolment and at previous screening) as free of $\geq \mathrm{CIN2}(\mathrm{n}=834) . \mathrm{P}_{\mathrm{McN}}=\mathrm{McNemar} \chi^{2}$ test; $\mathrm{P}_{\mathrm{n}}=$ matched non-inferiority test.

CIN2, cervical intraepithelial neoplasia grade 2; EIA, enzyme immunoassay; HPV, human papillomavirus.

plastics prior to retesting, and altering the optimal baseline settings for the assay (Cy5 10 000, FAM 100 000, Hex 30000 and ROX 100 000). Use of different baseline settings might have reduced the invalidity rate, but it was standardised in the study to ensure that manufacturer's instructions were followed. Limitation of the starting material restricted further analysis into the matter.

Here, we report that the Papilloplex HR-HPV in SurePath samples shows near perfect agreement with the comparator test GP5+/6+EIA for all hrHPV types individually. Moreover, our data indicate that Papilloplex test is non-inferior for clinical sensitivity for both $\geq \mathrm{CIN} 2$ and $\geq \mathrm{CIN} 3$ compared with GP5 +/6+EIA. Additionally, clinical specificity was measured by analysis of $834 \leq$ CIN1 (2x NILM) which shows that Papilloplex is non-inferior to GP5+/6+EIA. Absolute specificity based on extended outcome which included women with two consecutive negative cytology results and women with normal colposcopy or $\leq \mathrm{CIN} 1(\mathrm{~N}=935)$ was $81.7 \%$ (95\% CI $0.79 \%$ to $0.84 \%)$.

There are limited data on the clinical performance of Papilloplex. A previous study demonstrated similar sensitivity and specificity of Papillopex compared with two other clinically validated assays (RealTime HPV and HC2) on 500 Thinprep samples as based on the analysis of $87 \geq \mathrm{CIN} 2$ samples within the cohort, the assay had similar sensitivity and specificity to the comparator assays. ${ }^{11}$

One limitation of our study was that intralaboratory and interlaboratory reproducibility was not assessed within this panel. Another potential limitation is that use of archived specimens may include a disadvantage to tests evaluated years after sample collection. However, we do not observe such time effect in this study nor in previous VALGENT iterations. For example, Oštrbenk et al found non-inferiority between three different assays tested up to 8 years apart. ${ }^{30}$

In conclusion, the Papilloplex HR-HPV test is a simple, rapid HPV test with high clinical sensitivity and specificity for detection of $\geq \mathrm{CIN} 2$ and $\geq \mathrm{CIN} 3$. The test fulfils the international clinical accuracy criteria for use in cervical cancer screening on SurePath samples. As a cross-platform PCR based test with full genotyping, Papilloplex HR-HPV has promise for both cervical screening and epidemiological workstreams. Further data on the use of the test in clinical settings and in the widely used ThinPrep PreservCyt medium along with intralaboratory and interlaboratory reproducibility of Papilloplex HR-HPV of the assay is warranted.
Take home messages

- Novel single-tube PCR assay for human papillomavirus (HPV) genotyping is clinically validated according to international guidelines.

- Multiplex probe amplification technology can be used for HPV genotyping.

- There is need for robust clinically validated assays for cervical screening.

\section{Handling editor Runjan Chetty.}

Contributors MA coordinates the VALGENT framework. JHB and MA designed the VALGENT 4 protocol. KC and WGVQ co-ordinated testing within the laboratories. RB, $E A B$ and DME performed the laboratory work, $K C, R B, L X$ and MA analyzed the data. $\mathrm{KC}$ and $\mathrm{RB}$ drafted the manuscript. All authors were involved in writing the paper and had final approval of the submitted version. RB is the guarantor for the work.

Funding This study is investigator led but funded by Genefirst.

Competing interests $K C, R B$ and $E A B^{\prime} s$ institution has received research funding or gratis consumables to support research from the following commercial entities in the last 3 years: Cepheid, Euroimmun, GeneFirst, SelfScreen, Hiantis, Seegene, Roche, Abbott and Hologic. MA and LX are supported by the RISCC Network (Grant No. 847845), coordinated by the Free University of Amsterdam (The Netherlands), and the SME Instrument Grant GA 806551 (HPV OncoPredict), funded by the Horizon 2020 Programme of DG Research and Innovation, European Commission (Brussels, Belgium). Sciensano, the institute where MA and LX are employed, received support from Becton Dickinson, Genomica SAU, Agena Biotech Gmbh, Zhanghai Biotech (LifeRiver), GeneFirst and FujiRebio for methodological and statistical work as described in the VALGENT protocol (Arbyn et al, J Clin Virol 2016). VALGENT is a researcher induced study protocol where manufacturers can participate under the condition of providing test kits and covering research costs. Researchers do not receive any financial advantage by collaborating in VALGENT.

Patient consent for publication Not applicable.

Ethics approval The study was approved by the Danish Data Inspection Agency J. No. AHH-2017-024, I-Suite: 05356.

Provenance and peer review Not commissioned; externally peer reviewed.

Data availability statement Data are available on reasonable request.

Open access This is an open access article distributed in accordance with the Creative Commons Attribution Non Commercial (CC BY-NC 4.0) license, which permits others to distribute, remix, adapt, build upon this work non-commercially, and license their derivative works on different terms, provided the original work is properly cited, appropriate credit is given, any changes made indicated, and the use is non-commercial. See: http://creativecommons.org/licenses/by-nc/4.0/.

\section{ORCID iD}

Ramya Bhatia http://orcid.org/0000-0001-5294-0668

\section{REFERENCES}

1 Ronco G, Dillner J, Elfström KM, et al. Efficacy of HPV-based screening for prevention of invasive cervical cancer: follow-up of four European randomised controlled trials. Lancet 2014;383:524-32.

2 Arbyn M, Ronco G, Anttila A, et al. Evidence regarding human papillomavirus testing in secondary prevention of cervical cancer. Vaccine 2012;30 Suppl 5:F88-99.

3 Sankaranarayanan R, Nene BM, Shastri SS, et al. Hpv screening for cervical cancer in rural India. N Engl J Med 2009;360:1385-94.

4 Arbyn M, Simon M, Peeters E, et al. 2020 list of human papillomavirus assays suitable for primary cervical cancer screening. Clin Microbiol Infect 2021;27:1083-95.

5 Arbyn M, Snijders PJF, Meijer CJLM, et al. Which high-risk HPV assays fulfil criteria for use in primary cervical cancer screening? Clin Microbiol Infect 2015;21:817-26.

6 Poljak M, Kocjan BJ, Oštrbenk A, et al. Commercially available molecular tests for human papillomaviruses (HPV): 2015 update. J Clin Virol 2016;76 Suppl 1:S3-13.

7 download.pdf [Internet]. Available: https://www.fda.gov/media/122799/download [Accessed 09 Mar 2021].

8 Arbyn M, Anttila A, Jordan J, et al. European Guidelines for Quality Assurance in Cervical Cancer Screening. Second edition--summary document. Ann Oncol 2010;21:448-58

9 Fu G, Miles A, Alphey L. Multiplex detection and SNP genotyping in a single fluorescence channel. PLoS One 2012;7:e30340.

10 Sakellariou GK, Bilski M, Moreau M, et al. Principles and analytical performance of Papilloplex® HR-HPV, a new commercial CE-IVD molecular diagnostic test for the detection of high-risk HPV genotypes. Diagn Microbiol Infect Dis 2019;95:46-54. 
11 Bhatia R, Serrano I, Wennington $H$, et al. Evaluation of a novel single-tube method for extended genotyping of human papillomavirus. J Clin Microbiol 2018;56:e01687-17.

12 Meijer CJLM, Berkhof J, Castle PE, et al. Guidelines for human papillomavirus DNA test requirements for primary cervical cancer screening in women 30 years and older. Int J Cancer 2009;124:516-20.

13 Arbyn M, Depuydt C, Benoy I, et al.VALGENT: a protocol for clinical validation of human papillomavirus assays. J Clin Virol 2016;76 Suppl 1:S14-21.

14 Bonde J, Ejegod DM, Cuschieri K, et al. The Valgent4 protocol: robust analytical and clinical validation of $11 \mathrm{HPV}$ assays with genotyping on cervical samples collected in SurePath medium. J Clin Virol 2018;108:64-71.

15 Cuschieri K, Geraets DT, Moore C, et al. Clinical and analytical performance of the Onclarity HPV assay using the VALGENT framework. J Clin Microbiol 2015:53:3272-9.

16 Heard I, Cuschieri K, Geraets DT, et al. Clinical and analytical performance of the PapilloCheck HPV-Screening assay using the VALGENT framework. J Clin Virol 2016;81:6-11.

17 Cuschieri K, Geraets D, Cuzick J, et al. Performance of a Cartridge-Based assay for detection of clinically significant human papillomavirus (HPV) infection: lessons from VALGENT (validation of HPV genotyping tests). J Clin Microbiol 2016:54:2337-42.

18 Polman NJ, Oštrbenk A, Xu L, et al. Evaluation of the clinical performance of the HPVRisk assay using the VALGENT-3 panel. J Clin Microbio/ 2017;55:3544-51.

19 Viti J, Poljak M, Oštrbenk $A$, et al. Validation of EUROArray HPV test using the VALGENT framework. J Clin Virol 2018;108:38-42.

20 Oštrbenk A, Xu L, Arbyn M, et al. Clinical and analytical evaluation of the Anyplex II HPV HR detection assay within the VALGENT-3 framework. J Clin Microbiol. In Press 2018;56:e01176-18.
21 Xu L, Padalko E, Oštrbenk A, et al. Clinical Evaluation of INNO-LiPA HPV Genotyping EXTRA II Assay Using the VALGENT Framework. Int J Mol Sci 2018;19:2704.

22 Xu L, Oštrbenk A, Poljak M, et al. Assessment of the Roche linear array HPV genotyping test within the VALGENT framework. J Clin Virol 2018;98:37-42.

$23 \mathrm{Xu} \mathrm{L}$, Benoy I, Cuschieri K, et al. Accuracy of genotyping for HPV16 and 18 to triage women with low-grade squamous intraepithelial lesions: a pooled analysis of VALGENT studies. Expert Rev Mol Diagn 2019;19:543-51.

24 Heideman DAM, Xu L, Hesselink AT, et al. Clinical performance of the HPV-Risk assay on cervical samples in SurePath medium using the VALGENT-4 panel. J Clin Virol 2019;121:104201.

25 Ejegod DM, Lagheden C, Bhatia R, et al. Clinical validation of full genotyping CLART® HPV4S assay on SurePath and ThinPrep collected screening samples according to the International guidelines for human papillomavirus test requirements for cervical screening. BMC Cancer 2020;20:396.

26 Bhatia R, Boada EA, Bonde J, et al. Evaluation of HarmoniaHPV test for detection of clinically significant human papillomavirus infection using the VALGENT framework. J Virol Methods 2021;294:114161.

27 Heideman DAM, Hesselink AT, Berkhof J, et al. Clinical validation of the COBAS 4800 HPV test for cervical screening purposes. J Clin Microbiol 2011;49:3983-5.

28 Tang N-S, Tang M-L, Chan ISF. On tests of equivalence via non-unity relative risk for matched-pair design. Stat Med 2003;22:1217-33.

29 Cuschieri K, Wilson A, Palmer T, et al. The challenges of defining sample adequacy in an era of HPV based cervical screening. J Clin Virol 2021;137:104756.

30 Oštrbenk Valenčak A, Šterbenc A, Seme K, et al. Alinity M HR HPV assay fulfills criteria for human papillomavirus test requirements in cervical cancer screening settings. J Clin Microbiol 2019:58:e01120-19. 